\title{
A steradian of the southern sky at 151.5 MHz using the Mauritius Radio Telescope
}

\author{
V. N. Pandey and N. Udaya Shankar \\ Raman Research Institute, Bangalore 560 080, India \\ email: vnpandey,uday@rri.res.in
}

\begin{abstract}
We present a few results of a low-frequency southern sky survey carried out using the Mauritius Radio Telescope (MRT).
\end{abstract}

Keywords. catalogs, galaxies: clusters: general, surveys, supernova remnants

We present and discuss wide field images covering $\sim 1.25 \mathrm{sr}\left(18^{\mathrm{h}} \leqslant \alpha \leqslant 24^{\mathrm{h}},-75^{\circ} \leqslant \delta \leqslant\right.$ $-10^{\circ}$ ) of the sky at $151.5 \mathrm{MHz}$ with an angular resolution of $4^{\prime} \times 4.6$ and an rms noise of $260 \mathrm{mJy}$ beam $^{-1}$. A source catalog of $\sim 2800$ sources and its comparative study including cross identification with the Molonglo Reference Catalogue at $408 \mathrm{MHz}$ and Culgoora catalogue at $160 \mathrm{MHz}$ is described. The comparison reveals that there are no systematics in the final source list and the positional accuracies agree typically to about 0.07 times the MRT beam width. The procedures developed for flux calibration, which involves scaling different images to a common level, estimation of the primary beam shape of the helix, and recovery of the amplitude information of the signal lost in a 2-bit, 3-level correlator with an AGC are discussed. Our true flux density estimates agree to within $6.3 \%$ with the known flux densities of unresolved sources in the MS 4Jy sample.

Due to low-frequency and availability of short spacings, our images (surface brightness sensitivity $\sim 2.1 \times 10^{-21} \mathrm{~W} \mathrm{~m}^{-2} \mathrm{~Hz}^{-1} \mathrm{sr}^{-1}$ ) are sensitive to extended sources like giant radio sources, relics and supernova remnants (SNRs). Recently observed sub-structures in X-ray temperature maps support the existence of extended shock waves at locations of several known cluster relics. We show a specific example of such extended radio emission from these proposed shocks around the X-ray cluster Abell 3667 in the MRT image. These are steep spectrum sources, and we expect to detect quite a few such sources in the MRT survey.

An additional $0.6 \mathrm{sr}$ of the sky $\left(15^{\mathrm{h}} \leqslant \alpha \leqslant 18^{\mathrm{h}},-75^{\circ} \leqslant \delta \leqslant-10^{\circ}\right)$ has also been imaged and its comparison with existing low frequency images to search for new SNR candidates is under progress. As an example of interesting sources in the images, we briefly discuss a few steep spectrum sources, giant radio sources, double sources, fossil galaxies and report the structure of a few resolved SNRs.

\section{References}

Pandey, V. N., Oozeer, N., Udaya Shankar, N., \& Somanah, R. 2002, Bull. Astron. Soc. India, 30,773

Pandey, V. N., Udaya Shankar, N., \& Somanah, R. 2002, ApESS, 282, 29

Pandey, V. N., \& Udaya Shankar, N. 2005, URSI General Assembly, Proc. Session J 